OPEN ACCESS

Edited by:

Stefania Ceruti,

University of Milan, Italy

Reviewed by:

Zhouguang Wang,

Albert Einstein College of Medicine,

United States

Marta Boccazzi,

University of Milan, Italy

*Correspondence:

Xiaolu Zhang

xiaoluz2006@163.com

Liang Liu

enjoyyz@163.com

Specialty section:

This article was submitted to

Neuropharmacology,

a section of the journal

Frontiers in Pharmacology

Received: 28 March 2019

Accepted: 18 June 2019

Published: 16 July 2019

Citation:

Liu L, Zhao Z, Yin Q and Zhang X

(2019) TTB Protects Astrocytes Against Oxygen-Glucose Deprivation/

Reoxygenation-Induced Injury via Activation of Nrf2/HO-1 Signaling Pathway.

Front. Pharmacol. 10:792.

doi: 10.3389/fphar.2019.00792

\section{TTB Protects Astrocytes Against Oxygen-Glucose Deprivation/ Reoxygenation-Induced Injury via Activation of Nrf2/HO-1 Signaling Pathway}

\author{
Liang Liu ${ }^{1,2,3 *}$, Zhichen Zhao ${ }^{1}$, Qimeng Yin ${ }^{1}$ and Xiaolu Zhang ${ }^{4 *}$ \\ 1 Institute of Translational Medicine, Medical College, Yangzhou University, Yangzhou, China, ${ }^{2}$ Jiangsu Key Laboratory of \\ Experimental \& Translational Non-coding RNA Research, Yangzhou University, Yangzhou, China, ${ }^{3}$ Jiangsu Key Laboratory of \\ Zoonosis, Jiangsu Co-innovation Center for Prevention and Control of Important Animal Infectious Diseases and Zoonoses, \\ College of Veterinary Medicine, Yangzhou University, Yangzhou, China, ${ }^{4}$ Department of Pharmacy, Clinical Medical College, \\ Yangzhou University, Yangzhou, China
}

Neonatal hypoxic/ischemic encephalopathy $(\mathrm{NHIE})$ is a severe condition that leads to death or neurological disability in newborns. The underlying pathological mechanisms are unclear, and developing the target neuroprotective strategies are urgent. 2,7,2'-trihydroxy4,4'7'-trimethoxy-1,1'-biphenanthrene (TTB) is a natural product isolated from Cremastra appendiculata (D. Don) Makino and Liparis nervosa (Thunb.) Lindl. TTB has demonstrated potent cytotoxic activity against stomach (HGC-27) and colon (HT-29) cancer cell lines. However, none of the studies have addressed the effects of TTB in NHIE. In the present study, an oxygen-glucose deprivation/reoxygenation (OGD/R)-induced astrocyte injury model was established to investigate the effect of TTB and its potential mechanisms. Our results showed that TTB alleviated the OGD/R-induced reactive oxygen species increase and the intracellular antioxidant capacity of superoxide dismutase activity decrease. Moreover, TTB potentially prolonged the activation state of the nuclear factor erythroid 2-related factor 2 (Nrf2)/heme oxygenase-1 ( $\mathrm{HO}-1$ ) pathway and maintained the protection against oxidative stress in OGD/R-induced astrocytes by inducing the nuclear translocation and up-regulation of Nrf2 along with the enhanced expression of the downstream target gene $\mathrm{HO}-1$. Furthermore, TTB treatment diminished the accumulation of hypoxia-inducible factor-1 $\alpha(H I F-1 \alpha)$ and vascular endothelial growth factor (VEGF) expression induced by $\mathrm{OGD} / \mathrm{R}$. We also found TTB-treated astrocytes reversed the inhibition of OGD/R on neurite growth of neurons by the astrocyte-neuron coculture system. In conclusion, TTB inhibited the OGD/R-induced astrocyte oxidative stress at least partially through the inhibition of $\mathrm{HIF}-1 \alpha$ and VEGF via the Nrf2/HO-1 signaling pathway.

Keywords: neonatal hypoxia/ischemic encephalopathy, TTB, oxygen-glucose deprivation/reoxygenation, astrocytes, Nrf2/HO-1 signaling pathway, HIF-1 $\alpha$, VEGF 


\section{INTRODUCTION}

Neonatal hypoxia/ischemic encephalopathy (NHIE), also described as stroke in the neonatal period, is one of the most prevalent causes of a potentially devastating neonatal brain injury with long-term neurological deficits such as mental retardation, cerebral palsy, motor deficits, epilepsy, and learning and behavioral disabilities, which affects 1 to 8 of every 1000 live term births, with the highest rates in developing countries (Dilenge et al., 2001; Kurinczuk et al., 2010). The cases of infantile cerebral palsy are caused by the same factors that cause adult cerebral palsy (Nelson, 2007). At present, therapeutic hypothermia protocols are formally endorsed treatments, which significantly improve outcomes by leading to delayed cell death. However, its effectiveness is limited in severe cases, as $40 \%$ to $50 \%$ of children with NHIE still die or suffer from long-term neurological disorders (Edwards et al., 2010). There are no effective pharmacological interventions available. To reduce the neurological consequences of NHIE, new and effective neuroprotective strategies are urgently needed.

Astrocytes are the largest population of glial cells in the brain and have been implicated in many functions as key mediators in the central nervous system (CNS). Astrocytes are highly involved in neuronal migration, adaptive plasticity, and synaptogenesis in the developing brain (Ullian et al., 2001; Guizzetti et al., 2014). The developing neonatal brain is particularly vulnerable to oxidative stress based on the immature free radical scavenging systems (Zorec et al., 2018). Several evidences have identified that NHIE causes long-lasting oxidative stress, a process aggravated by mitochondrial dysfunction. Reactive oxygen species (ROS) have been involved in the pathogenesis of NHIE and induce cell death via the oxidation of membrane lipids and proteins (Fatemi et al., 2009). Recently, a study demonstrated that astrocytes are a major source of increased brain ROS production during neonatal asphyxia (Parfenova et al., 2018).

Nuclear factor erythroid 2-related factor 2 (Nrf2) is a member of the basic region/leucine zipper transcription factor family that regulates several antioxidant pathways (Sandberg et al., 2014). Under unstressed conditions, Nrf2 is binding to the homodimeric protein Kelch-like ECH-associated protein 1 (Keap1), which becomes the Nrf2 Keap1 complex in the cytoplasm. In pathological processes, such as oxidative stress and other insult attacks, Nrf2 is activated by release from the antioxidant response element of Keap1 and translocated to the nucleus from the cytoplasm, which leads to accumulate in the nucleus, regulates genetic activities, and induces cytoprotective action (Cao et al., 2015). Nrf2 activation drives several functions, including

\footnotetext{
Abbreviations: NHIE, neonatal hypoxic/ischemic encephalopathy; TTB, 2,7,2'-trihydroxy-4,4'7'-trimethoxy-1,1'-biphenanthrene; OGD/R, oxygen-glucose deprivation/reoxygenation; CNS, central nervous system; ROS, reactive oxygen species; SOD, superoxide dismutase; LDH, lactate dehydrogenase; Nrf2, nuclear factor erythroid 2-related factor 2; HO-1, heme oxygenase-1; HIF-1a, hypoxiainducible factor-1 $\alpha$; VEGF, vascular endothelial growth factor; GFAP, glial fibrillary acidic protein; Keap1, Kelch-like ECH-associated protein 1; DMEM, high-glucose Dulbecco's modified Eagle medium; MTT, 3-(4,5-dimethyl-2thiazolyl)-2,5-diphenyl-2H-tetrazolium bromide; H/I, hypoxia/ischemia.
}

antioxidative stress, antiapoptosis, and anti-inflammation, via several molecules and pathways (Shu et al., 2016).

$2,7,2^{\prime}$-trihydroxy-4,4'7' -trimethoxy-1,1'-biphenanthrene (TTB) is a biphenanthrene isolated from Cremastra appendiculata (D. Don) Makino (Xue et al., 2006; Liu et al., 2016a) and Liparis nervosa (Thunb.) Lindl. (Liu et al., 2016b), which both belong to the family Orchidaceae. Research concerning bioactivities of TTB was very limited, and it was only reported to have cytotoxic activity against stomach (HGC-27) and colon (HT-29) cancer cell lines (Liu et al., 2016b). Therefore, it is necessary to explore other bioactivities of TTB.

Oxygen-glucose deprivation/reoxygenation (OGD/R) is a widely used cell model to mimic the aspects of cell death observed in a hypoxia brain injury model, including neonate HI and adult ischemic stroke (Cengiz et al., 2014; Tasca et al., 2015). Several recent studies demonstrated that Nrf2 was regulated by special compounds in the rat neonatal $\mathrm{HI}$ brain injury model (Cui et al., 2017; Gao et al., 2018). We speculated that TTB may offer neuroprotection in part by regulating Nrf2 in reactive astrocytes. In the current study, we investigated the effect of TTB on the OGD/R-induced astrocyte injury model, which is to mimic NHIE in vitro. We observed that Nrf2 activation via TTB treatment improved astrocyte function by targeting oxidative stress. Our findings suggested that astrocytic Nrf2 could be a potential therapeutic target for the treatment of NHIE.

\section{MATERIALS AND METHODS}

\section{Compound}

The ethyl acetate extract of $L$. nervosa (Thunb.) Lindl. was isolated and purified using repeated column chromatography over Sephadex LH-20, RP-C18, silica gel, and semi-preparative high performance liquid chromatography (HPLC) to obtain TTB. The purity of TTB was at least $99 \%$ as judged by HPLC analysis. All the extraction, separation, and purification were performed by our group (Liu et al., 2016b).

\section{Cell Culture}

Postnatal day 1 Sprague-Dawley rats were purchased from the Comparative Medicine Center of Yangzhou University (Yangzhou, China) and used for culturing astrocytes as described previously (Hertz et al., 1998; Zhang et al., 2014). Briefly, the cerebral cortex was taken in a sterile environment and then dispersed with $0.25 \%$ trypsin (Gibco Co., Grand Island, NY, USA) for $10 \mathrm{~min}$ at $37^{\circ} \mathrm{C}$. The cells were plated in $75 \mathrm{~cm}^{2}$ flasks precoated with $40 \mu \mathrm{g} / \mathrm{ml}$ poly-D-lysine, grown in high-glucose Dulbecco's modified Eagle medium (DMEM; Gibco Co.) containing 10\% fetal bovine serum (FBS; Gibco Co)., 100 units $/ \mathrm{ml}$ penicillin, and $100 \mu \mathrm{g} / \mathrm{ml}$ streptomycin (Solarbio, Beijing, China), and placed in an incubator at 5\% $\mathrm{CO}_{2}, 95 \%$ air at $37^{\circ} \mathrm{C}$. The flasks were gentle shaken about 150 times by hand to remove the layer of nonadherent cells growing on the top of the flat monolayer when changing the medium every 2 to 3 days. More than $95 \%$ astrocytes were achieved by 
the cultures. After 14 days in culture, astrocytes were grown to confluence and then plated in the appropriate vessel. When the cultures reached $70 \%$ to $80 \%$ confluence, cells were ready for treatment.

\section{Establishment of OGD/R-Induced Injury of Astrocytes}

The OGD/R model was established in astrocytes. Briefly, the cells were washed twice with phosphate-buffered saline (PBS) and incubated in glucose- and FBS-free medium and then placed in an anoxic incubator at $94 \% \mathrm{~N}_{2}, 1 \% \mathrm{O}_{2}, 5 \% \mathrm{CO}_{2}$ at $37^{\circ} \mathrm{C}$. After OGD for $6 \mathrm{~h}$, the medium was changed back to high-glucose DMEM containing $10 \%$ FBS and returned to the normal oxygen incubator for another $24 \mathrm{~h}$. The blank control group in the experiment was always kept in a normal oxygen incubator and cultured in high-glucose medium containing 10\% FBS. The cells were given OGD/R treatment with or without TTB (1.5625, 6.25 , and $25 \mu \mathrm{M})$. For certain experiments, the astrocytes were preincubated in ML385 (Nrf2-specific inhibitor) for $12 \mathrm{~h}$ before $\mathrm{OGD} / \mathrm{R}$.

\section{Cell Viability}

Primary astrocytes were incubated into 96-well plates at a density of about $1 \times 10^{4}$ cells per well. Cell viability was estimated using 3-(4,5-dimethyl-2-thiazolyl)-2,5-diphenyl-2H-tetrazolium bromide (MTT; Solarbio) and lactate dehydrogenase (LDH) assay kit (Nanjing Jiancheng Bioengineering Institute, Nanjing, China). The optical absorbance was read on a plate reader at a wavelength of $490 \mathrm{~nm}$ for MTT. LDH release from damaged cell membrane was indicated as a percentage of total LDH according to the manufacturer's instruction.

\section{Measurement of Superoxide Dismutase (SOD) Levels}

The SOD activity in the astrocytes was measured by a commercially available kit (Nanjing Jiancheng Bioengineering Institute) according to the manufacturer's instruction. Briefly, after treatment, cells were washed with cold PBS twice and collected. The homogenates were centrifuged for $10 \mathrm{~min}$ at 10,000 $\mathrm{rpm}$ at $4^{\circ} \mathrm{C}$ and supernatants were used for SOD activities. The optical absorbance was read on a plate reader at a wavelength of $450 \mathrm{~nm}$. The protein concentration was determined by BCA assay.

\section{Intracellular ROS Assay}

Astrocytes were seeded in 96-well plates at a density of $1 \times$ $10^{4}$ cells per well. After exposure to OGD/R, the medium with different concentrations of TTB was replaced with $2^{\prime}, 7^{\prime}$-dichlorodihydrofluorescein diacetate $(10 \mu \mathrm{M})$ in DMEM. The cells were incubated at $37^{\circ} \mathrm{C}$ for $30 \mathrm{~min}$ in the dark and then washed twice with PBS. The fluorescence was tested on a microplate reader using excitation/emission wavelengths $(\mathrm{Ex} / \mathrm{Em})$ of $488 / 525 \mathrm{~nm}$.

\section{Western Blot Analysis}

Total proteins were extracted with lysis radioimmunoprecipitation assay buffer (Applygen, Beijing, China) and protease inhibitor cocktail (Applygen). The protein concentrations were determined by BCA assay (Beyotime, Shanghai, China). All steps were carried out on ice. Nuclear and cytosolic proteins were extracted using a commercial kit (KeyGEN BioTECH's, Nanjing, China). The extracts were boiled in a metal bath at $95^{\circ} \mathrm{C}$ for $5 \mathrm{~min}$. Subsequently, sodium dodecyl sulfate-polyacrylamide gel electrophoresis was carried out to separate the proteins. The proteins were then transferred to a polyvinylidene fluoride (PVDF; Solarbio) membrane for about $1.5 \mathrm{~h}$. After blocking in 5\% nonfat milk (Applygen) for $2 \mathrm{~h}$, the PVDF membrane was incubated with the primary antibodies antiNrf2 (1:500; Santa Cruz Biotechnology, Santa Cruz, CA, USA), antiheme oxygenase-1 (HO-1; 1:500; Wanlei Biotechnology, Shenyang, China), anti-hypoxia-inducible factor-1 $\alpha$ (HIF-1 $\alpha$; 1:500; BBI, Shanghai, China), anti- $\beta$-actin (1:5,000; abclonal, Wuhan, China), and anti-lamin B (1:500; abclonal) overnight at $4^{\circ} \mathrm{C}$ then followed by horseradish peroxidase-conjugated secondary antibodies for $2 \mathrm{~h}$ at room temperature. The membranes were washed three times for 10 min before obtaining protein bands by enhanced chemiluminescence reagents (Beyotime) and analyzed by ImageJ.

\section{Immunofluorescence Assay}

The astrocytes were fixed with $4 \%$ paraformaldehyde for 30 min and permeabilized with $0.1 \%$ Triton X-100 (Solarbio) for $10 \mathrm{~min}$ at room temperature. After blocking with $3 \%$ bovine serum albumin for $30 \mathrm{~min}$ at room temperature, the cells were incubated with the primary antibody anti-Nrf2 (1:200; Santa Cruz Biotechnology) at $4^{\circ} \mathrm{C}$ overnight followed by Alexa Fluor 488 donkey anti-mouse antibody or Alexa Fluor 594 donkey anti-rabbit antibody (1:500; Invitrogen, Carlsbad, CA, USA). Nuclei were stained by 4',6-diamidino-2-phenylindole (DAPI; $0.5 \mu \mathrm{g} / \mathrm{ml}$; Beyotime), and images were acquired using a Zeiss fluorescence microscope attached to a digital camera.

\section{Real-Time Polymerase Chain Reaction (PCR)}

Total RNA was extracted using Trizol reagent and dissolved in ultrapure distilled water (Invitrogen). Equal amounts of RNA were reverse transcribed at $25^{\circ} \mathrm{C}$ for $5 \mathrm{~min}, 42^{\circ} \mathrm{C}$ for $60 \mathrm{~min}$, and $70^{\circ} \mathrm{C}$ for 5 min using RevertAid First Strand cDNA Synthesis Kit (Thermo Fisher Scientific, Waltham, MA, USA). cDNA amplification was carried out in $20 \mu \mathrm{l}$ PCR buffer using AceQ quantitative PCR (qPCR) SYBR Green Master Mix (Vazyme, Nanjing, China). The primers used for amplification in the experiment were as follows: HIF-1a sense $5^{\prime}$-GTCTCCATTACCTGCCTCTG-3' and antisense $5^{\prime}$-GATTCTTCGCTTCTGTGTCTTC-3' ${ }^{\prime}$, vascular endothelial growth factor (VEGF) sense $5^{\prime}$-ACCCCACAAAG AGCTAGATAG-3' and antisense $5^{\prime}$-CCTCTTCACTAAATGAC AGTCCC- $3^{\prime}$, and glial fibrillary acidic protein (GFAP) sense 5'-CCTTGCGCGGCACGAACGAG-3' and antisense 5'-CCGA GCGAGTGCCTCCTGGT-3'. mRNA levels were normalized 
to levels of $\beta$-actin measured in the same samples (sense 5'-GCGTCCACCCGCGAGTACAA-3' and antisense 5' -TCCA TGGCGAACTGGTGGCG-3').

\section{Astrocyte-Neuron Coculture}

The astrocytes were passed in glass coverslips placed into 24 -well plate at cell density of $1 \times 10^{5}$ astrocytes per coverslip. When the cultures reached $70 \%$ to $80 \%$ confluence, the cells were subjected to OGD for $6 \mathrm{~h}$ and reoxygenation for $24 \mathrm{~h}$; at the same time, primary neurons were extracted. After OGD/R in astrocytes was completed, primary neurons were seeded at a concentration of $1.2 \times 10^{4}$ cells per well above the astrocytes and cocultured with DMEM for $24 \mathrm{~h}$.

\section{Statistical Analysis}

Statistical analyses were performed using Prism 5 software (GraphPad Software, Inc., San Diego, CA, USA). Data were expressed as the mean \pm standard error (SE) of at least three independent experiments and compared using one-way analysis of variance with Tukey's test. $p<0.05$ was considered statistically significant difference.

\section{RESULTS}

\section{TTB Attenuated OGD/R-Induced Damage in Astrocytes}

To examine the cell toxicity of TTB and the protective effect of TTB against cytotoxicity induced by OGD/R, the MTT assay was used to assess the viability of astrocytes. There was no cytotoxicity in the TTB concentration range from 1.5625 to $50 \mu \mathrm{M}$ (Figure 1A). The viability of astrocytes exposed to OGD/R was significantly decreased compared to the blank control group, but this effect was reversed after treatment with TTB at concentrations of 1.5625 , 6.25 , and $25 \mu \mathrm{M}$ (Figure 1B). These results indicated that TTB treatment was noncytotoxic and TTB attenuated OGD/R-induced astrocyte damage. Exposure to OGD/R significantly increased the release of $\mathrm{LDH}$, whereas treatment with TTB markedly reduced the OGD/R-induced LDH release in astrocytes (Figure 1C).

\section{TTB Alleviated OGD/R-Induced Oxidative Stress in Astrocytes}

To examine the effect of TTB treatment on OGD/R-induced oxidative stress in astrocytes, we examined the SOD activity and the ROS level. We found that treatment with TTB significantly

A

B
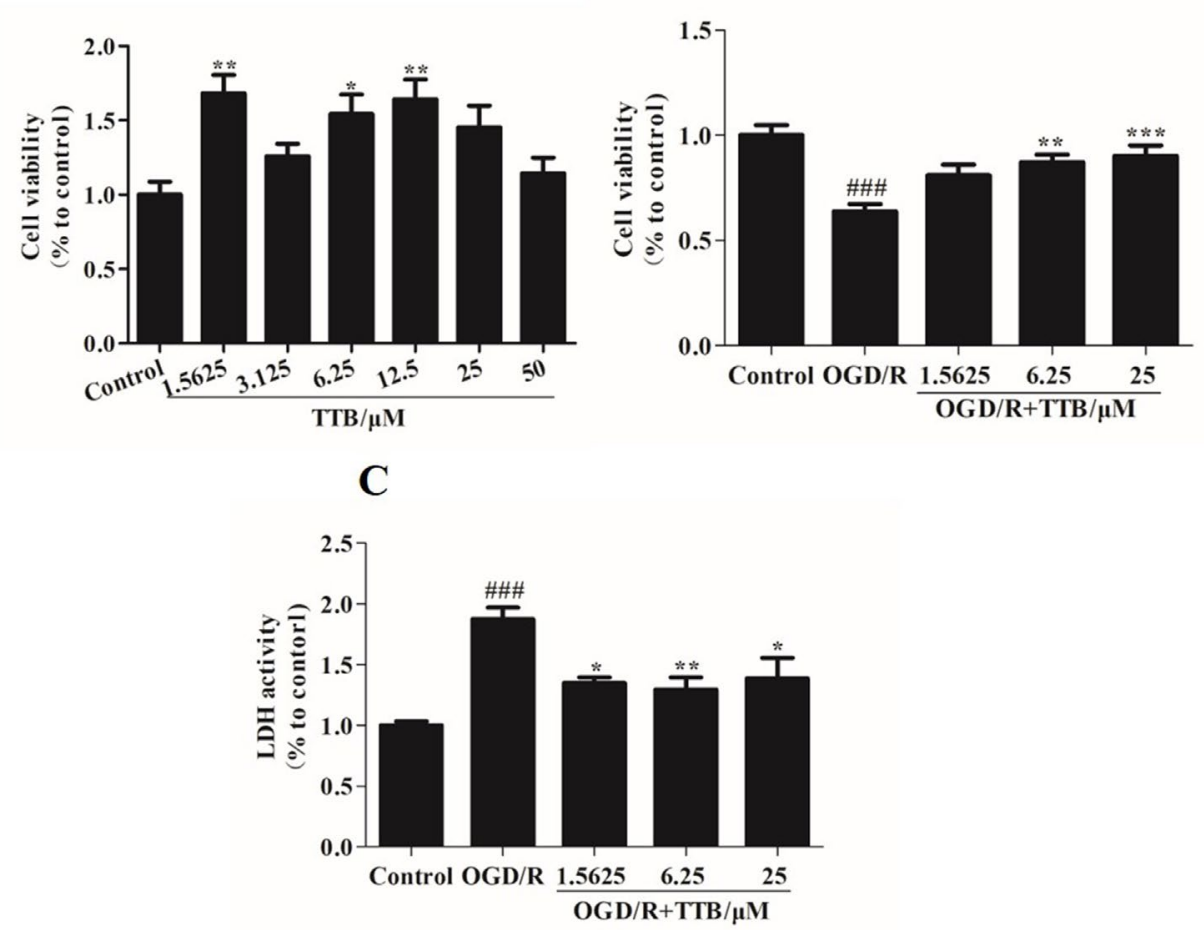

FIGURE 1 | Cell toxicity of TTB in regular astrocytes and effect of TTB on cell viability under oxygen-glucose deprivation/reoxygenation (OGD/R) were evaluated. (A) Primary astrocytes were incubated with TTB at the concentration range from 1.5625 to $50 \mu \mathrm{M}$ for $30 \mathrm{~h}$ in the normal incubator. Cell survival was estimated by the MTT assay. (B and $\mathbf{C}$ ) Primary astrocytes were incubated with TTB at 1.5625, 6.25, and $25 \mu \mathrm{M}$ for $6 \mathrm{~h}$ OGD and $24 \mathrm{~h}$ reoxygenation. Cell survival and cell death were estimated by the MTT and LDH assays, respectively. ${ }^{\star} p<0.05,{ }^{* \star} p<0.01$ vs. blank control group; ${ }^{\# \# \#} p<0.001$ vs. blank control group; ${ }^{\star} p<0.05$, ${ }^{\star \star} p<0.01$, ${ }^{\star \star \star} p<0.001$ vs. OGD/R group. Data are mean \pm SE of three independent experiments. 
reversed the decrease of SOD activity and the increase of intracellular ROS due to OGD/R (Figures 2A, B). These results indicated that TTB significantly improved OGD/R-induced oxidative stress in astrocytes.

\section{TTB Induced Nrf2 Up-Regulation and Nuclear Translocation in OGD/R-Injured Astrocytes}

When a stress response occurs, intracellular Nrf2 is easily transferred to the nucleus from the cytoplasm, which subsequently initiates the transcriptional activation of various antioxidant enzymes and phase II detoxification enzymes. The effect of TTB on Nrf2 expression and nuclear translocation in OGD/R-induced astrocytes was determined by Western blot and immunofluorescence. Lamin $\mathrm{B}$ was used to assess the purity of the nuclear fraction. As shown in Figure 3A, compared to the control group, Nrf2 protein expression was dramatically increased by OGD for $6 \mathrm{~h}$ and reoxygenation for $24 \mathrm{~h}$ but decreased by 12 and $24 \mathrm{~h}$ OGD and $24 \mathrm{~h}$ reoxygenation. TTB at $6.25 \mu \mathrm{M}$ up-regulated Nrf2 protein expression in all three time courses under OGD/R. Moreover, OGD for $6 \mathrm{~h}$ and reoxygenation for $24 \mathrm{~h}$ significantly increased $\mathrm{Nrf} 2$ nuclear translocation in astrocytes, and treatment with TTB at 1.5625, 6.25, and $25 \mu \mathrm{M}$ further facilitated Nrf2 translocation to the nucleus compared to the OGD/R group (Figures 3B-D). These results indicated that TTB treatment could up-regulate Nrf2 expression and promote Nrf2 nuclear translocation under OGD/R condition.

\section{TTB Activates the Nrf2/HO-1 Pathway}

$\mathrm{Nrf} 2$ is activated under stress conditions and translocates to the nucleus to initiate transcriptional activation of HO-1. Therefore, we examined the effect of TTB on the expression of Nrf2 and HO-1 proteins in astrocytes by Western blot. Cells were incubated with TTB at $1.5625,6.25$, and $25 \mu \mathrm{M}$ under OGD for $6 \mathrm{~h}$ and reoxygenation for $24 \mathrm{~h}$. As a result, we found that, compared to the OGD/R group, Nrf2 and $\mathrm{HO}-1$ expression in protein level was significantly up-regulated in the OGD/R+TTB group (Figures 4A, B), which indicated that TTB might get involved in the Nrf2/HO-1 signal pathway.

\section{TTB Inhibited OGD-Induced HIF-1 $\alpha$ Accumulation, VEGF Release, and GFAP Expression in Astrocytes}

OGD/R injury could trigger HIF-1a up-regulation. Nrf2 and HIF- $1 \alpha$ are two transcription factors that represent oxygen and redox state. We further observed the effect of TTB on HIF-1a expression in both protein and mRNA levels under OGD/R condition. Cells were treated with TTB in different concentrations $(1.5625,6.25$, and $25 \mu \mathrm{M})$ for $6 \mathrm{~h}$ OGD and $24 \mathrm{~h}$ reoxygenation in astrocytes. The results showed that TTB treatment inhibited OGD/R-induced up-regulation of HIF-1a (Figures 5A, B). Moreover, VEGF and GFAP gene expressions were measured by qPCR. TTB inhibited OGD/R-induced increase in GFAP and VEGF gene expressions (Figures 5C, D).

\section{TTB Prevented OGD/R-Induced Inhibition of Neurite Outgrowth in Neuron-Astrocyte Coculture System}

To test the hypothesis that OGD-treated astrocytes inhibit neurite outgrowth and whether TTB could alleviate the inhibition, we plated the neurons on top of the OGD/R-treated astrocytes in the presence of TTB at a concentration of $6.25 \mu \mathrm{M}$. We observed that neurons cocultured with OGD/R-induced astrocytes developed shorter major and minor neurites compared to neurons cocultured with the control astrocytes, whereas TTB attenuated the OGD/Rinduce inhibition of neurite growth. (Figure 6A-D). This result indicated that TTB could regulate astrocyte function and promote neuronal growth.

\section{Nrf2 Inhibitor Abolished the Protective Effect of TTB by the Nrf2/HO-1 Pathway in OGD/R-Injured Astrocytes}

To investigate whether Nrf2 function contributes to the neuroprotective effects of TTB, astrocytes were incubated with ML385, the Nrf2-specific inhibitor, to inhibit Nrf2 expression. Under OGD/R treatment, the expression of Nrf2 and HO-1 was inhibited by ML385 at a concentration of $5 \mu \mathrm{M}$. ML385 also
A

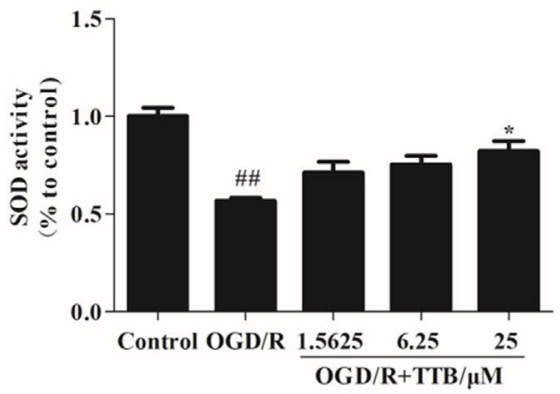

B

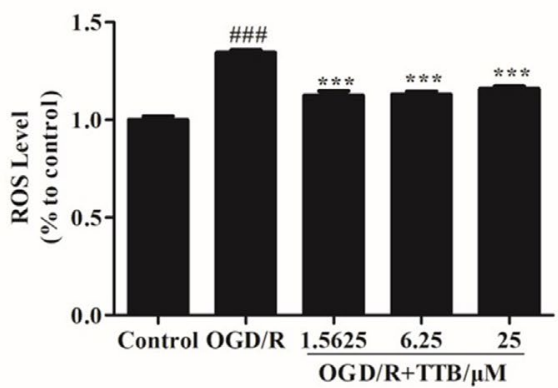

FIGURE 2 | TTB inhibited the oxidative stress in astrocytes under OGD/R exposure. Primary astrocytes were treated with OGD for $6 \mathrm{~h}$ and reoxygenation for $24 \mathrm{~h}$ in the presence of TTB at 1.5625, 6.25, and $25 \mu \mathrm{M}$. (A) SOD activity was determined by the SOD assay. (B) ROS levels were estimated by the ROS assay. \#\# $p<0.01$,

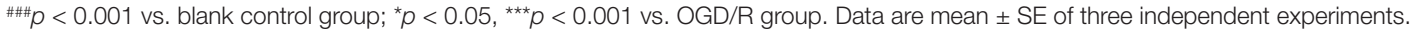


A
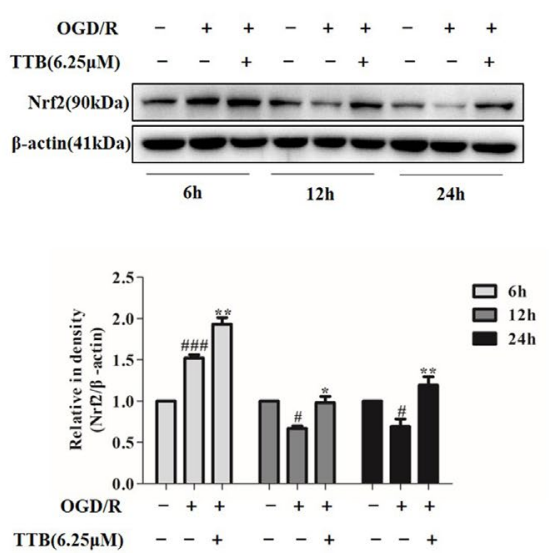

D
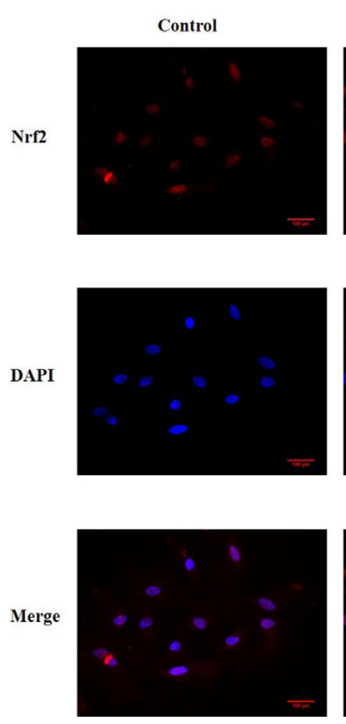

B

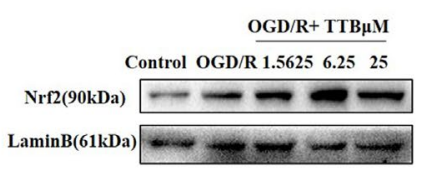

C
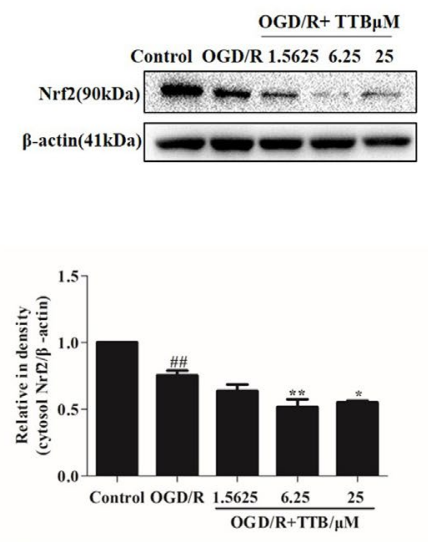
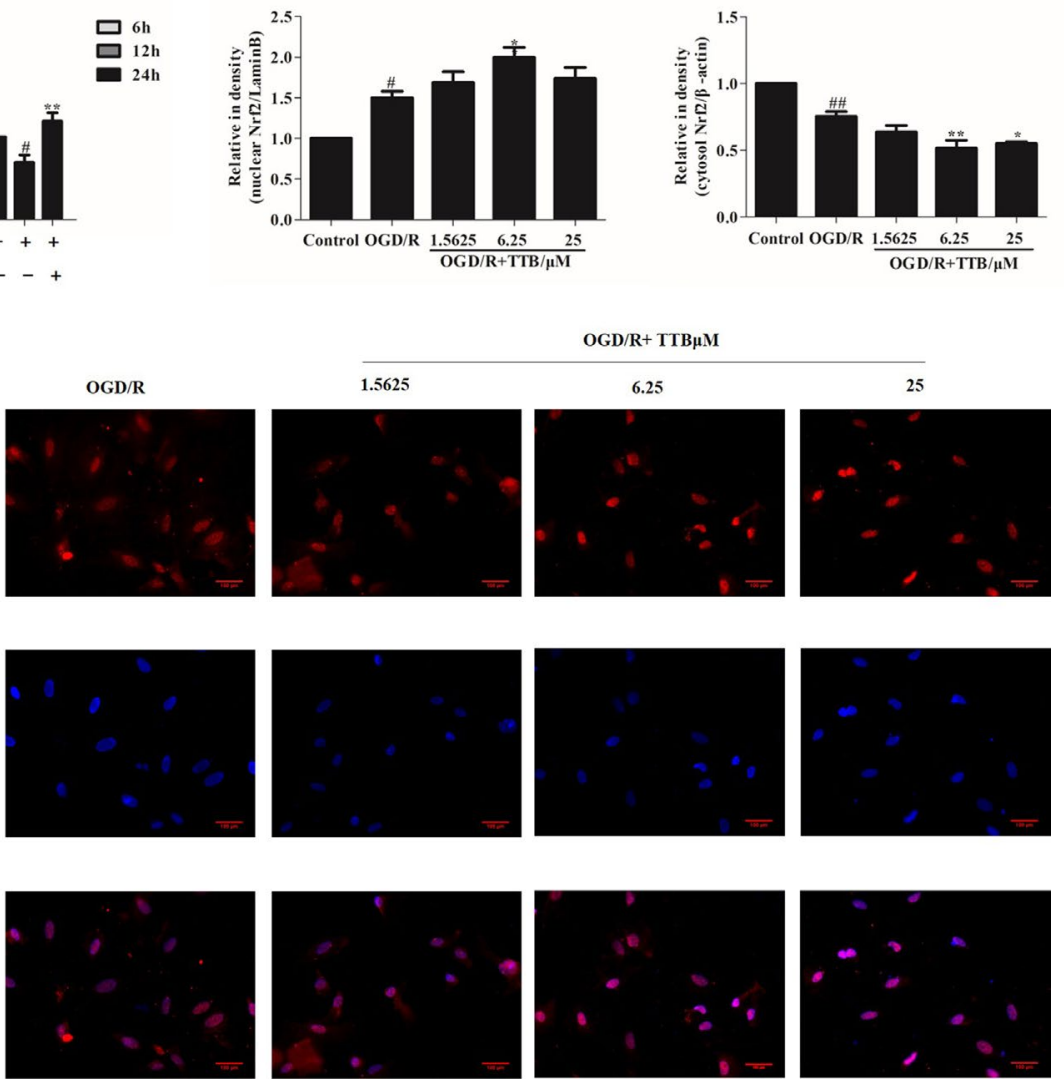

FIGURE 3 | TTB induced Nrf2 activation and nuclear translocation in OGD/R-injured astrocytes. Primary astrocytes were incubated with TTB under OGD/R. Total proteins from treated astrocytes were extracted and used for Western blot. Nuclear and cytosolic proteins were determined by Western blot and immunofluorescence. (A) TTB at $6.25 \mu \mathrm{M}$ increased Nrf2 expression in total protein under OGD for 6,12 , and $24 \mathrm{~h}$ after $24 \mathrm{~h}$ reoxygenation. (B and C) TTB at 1.5625, 6.25, and $25 \mu \mathrm{M}$ decreased Nrf2 expression in the cytosol of astrocytes but increased in the nucleus at $6 \mathrm{~h}$ OGD and $24 \mathrm{~h}$ rexoygenation. Data are presented as relative density units normalized to $\beta$-actin. (D) Immunofluorescence staining was performed to detect the effects of TTB on Nrf2 translocation at $6 \mathrm{~h}$

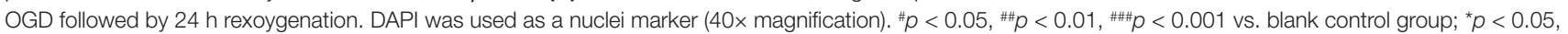
${ }^{* \star} p<0.01$ vs. OGD/R group. Data are mean \pm SE of three independent experiments.

abolished TTB-induced increase in Nrf2 and HO-1 expression (Figures 7A-C). Moreover, the effect of TTB on the expression of HIF- $1 \alpha$ and VEGF was inhibited by ML385 (Figures 7A, D, E). These data demonstrated that the neuroprotective effect of TTB may be through the activation of the Nrf2/HO-1 pathway.

\section{DISCUSSION}

NHIE causes a series of oxidative bursts, cell apoptosis, and cascade of inflammatory responses. The potential therapy strategies have been limited and unsatisfactory (Mulkey et al., 2011; Zalewska et al., 2015). Astrocyte dysfunction is critically involved in oxidative stress, apoptosis, and inflammation in the pathologic process of NHIE. The current studies were undertaken to identify the hypothesis that TTB exposure would initiate the protective response against OGD/R-induced injury in astrocytes, which is an in vitro model to mimic NHIE. The transcription factor Nrf2 was identified to play an important role in modulating the neuroprotective effects of TTB.

TTB is a natural biphenanthrene that is a relatively rare secondary metabolite in the plant kingdom. TTB was only reported to have cytotoxicity against HGC-27 and HT-29 cancer cell lines (Liu et al., 2016b). TTB contains phenolic 
A

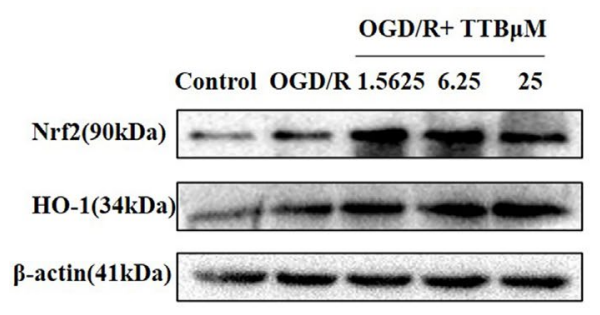

B

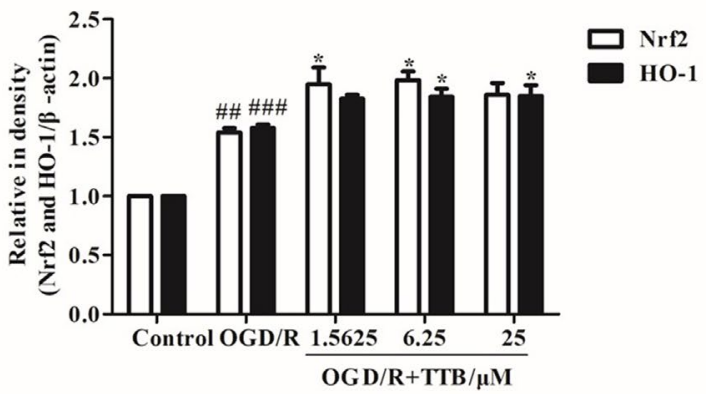

FIGURE 4 | TTB treatment activated Nrf2/HO-1 expression. Primary astrocytes were incubated with TTB at 1.5625, 6.25 , and $25 \mu \mathrm{M}$ for $6 \mathrm{~h}$ OGD and $24 \mathrm{~h}$ reoxygenation. (A and $\mathbf{B}$ ) Western blot was carried out to determine the expression of Nrf2 and HO-1, respectively. Data are presented as relative

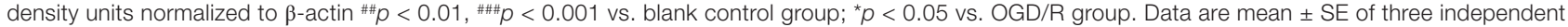
experiments.

A

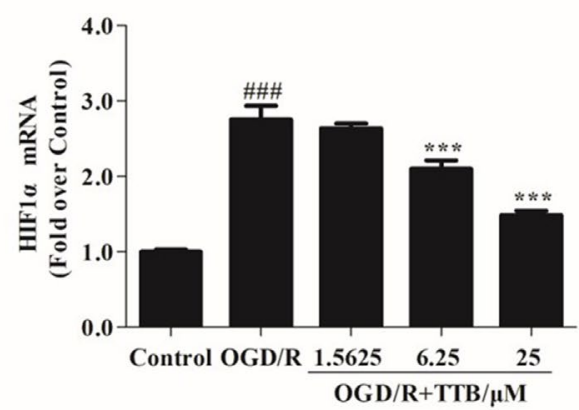

C

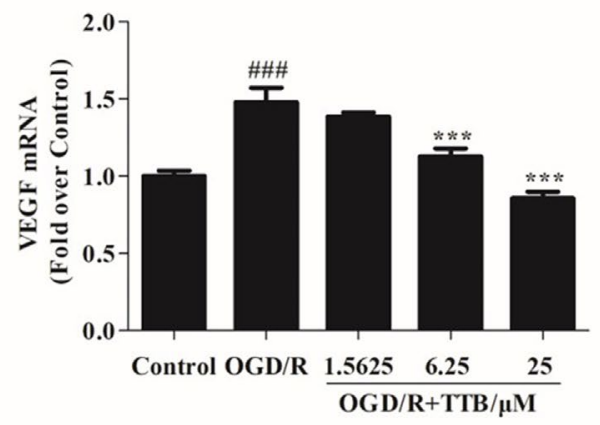

B

OGD/R+ TTB $\mu \mathrm{M}$

Control OGD/R $\overline{1.5625} \quad 6.25 \quad 25$

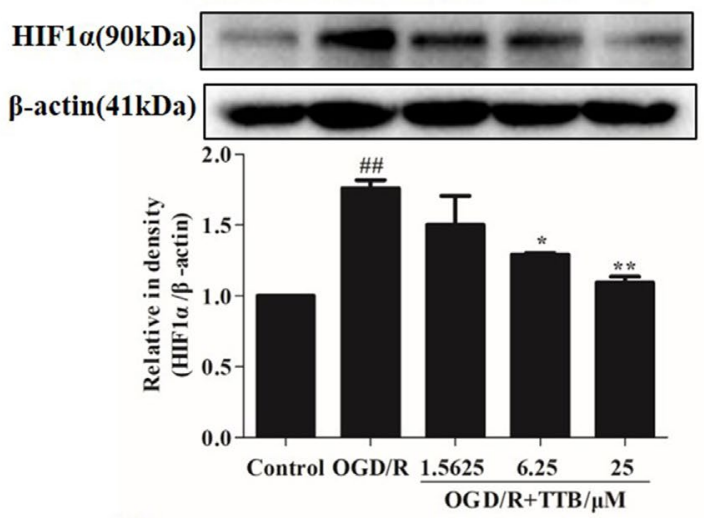

D

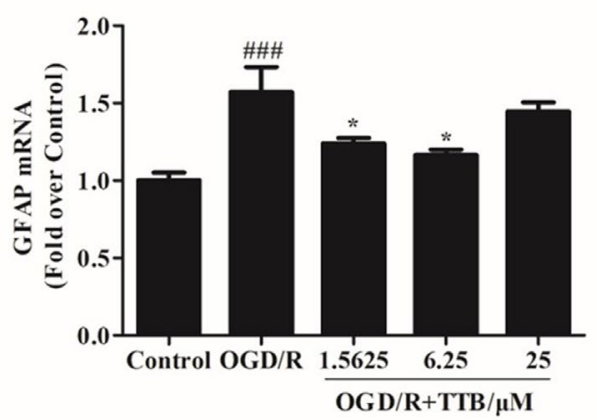

FIGURE 5 | TTB reduced HIF-1 $\alpha$, VEGF, and GFAP expression in OGD/R-injured astrocytes. Primary astrocytes were incubated with TTB at $1.5625,6.25$, and $25 \mu \mathrm{M}$ for $6 \mathrm{~h}$ OGD and $24 \mathrm{~h}$ reoxygenation. RNA was extracted and HIF- $1 \alpha$, VEGF, and GFAP mRNA levels were quantified by qPCR. Western blot was carried out for HIF-1 $\alpha$ protein determination. Results were normalized to $\beta$-actin and expressed as fold over control. (A and B) HIF- $1 \alpha$ mRNA expression was reduced by TTB treatment in OGD/R-injured astrocytes, which was consistent with HIF-1 $\alpha$ protein level. (C and D) TTB inhibited VEGF and GFAP mRNA expression in OGD/R-

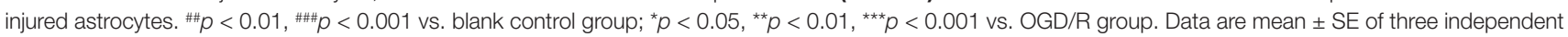
experiments. 
A

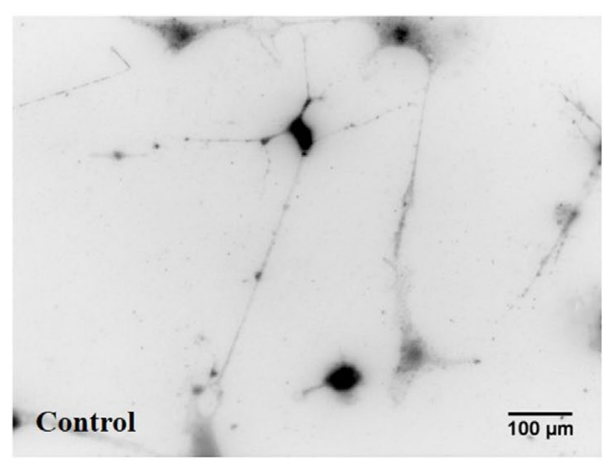

C

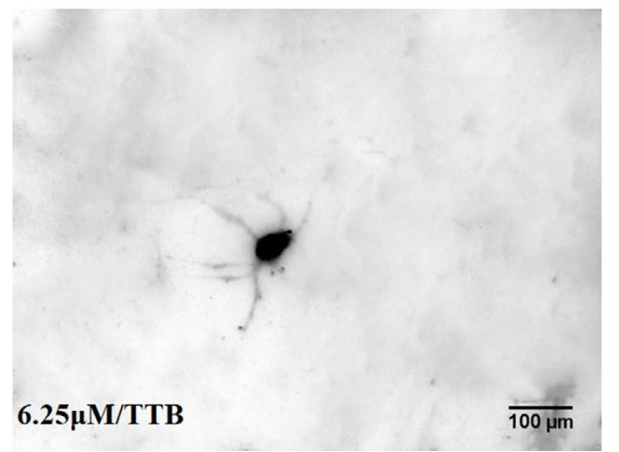

B

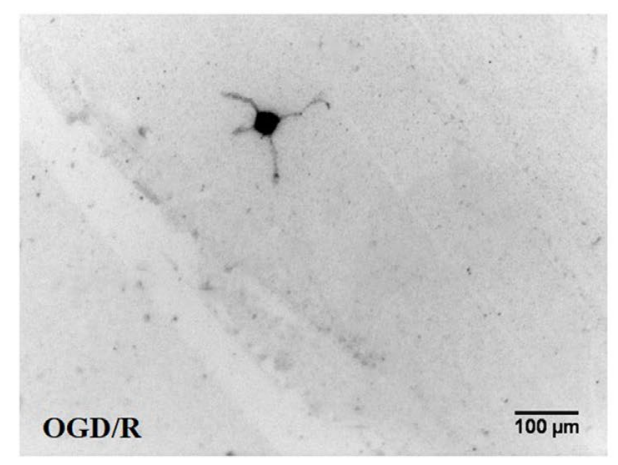

D

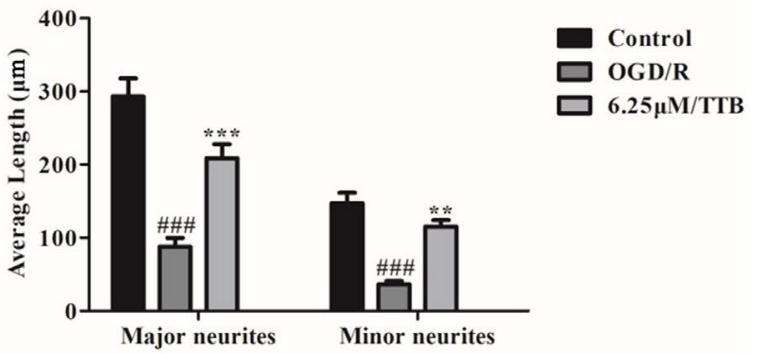

FIGURE 6 | Effects of TTB-treated astrocytes on hippocampal neuron neurite outgrowth. Astrocytes were treated with $6.25 \mu \mathrm{M} T \mathrm{~TB}$ for $6 \mathrm{~h}$ OGD and $24 \mathrm{~h}$ reoxygenation. Hippocampal neurons were plated on top of pretreated astrocytes for an additional $24 \mathrm{~h}$. Then, cultures were fixed and stained with the antibody of neuron-specific $\beta$-III-tubulin and a fluorescent secondary antibody. ImageJ was used to measure neurite length. (A) Control, (B) OGD/R, (C) TTB treatment, and (D) morphometric quantification of major neurite and minor neurite length. ${ }^{\# \# \#} p<0.001$ vs. blank control group; ${ }^{\star \star} p<0.01,{ }^{\star \star \star} p<0.001$ vs. OGD/R group. Data are mean \pm SE of three independent experiments.

\section{A}

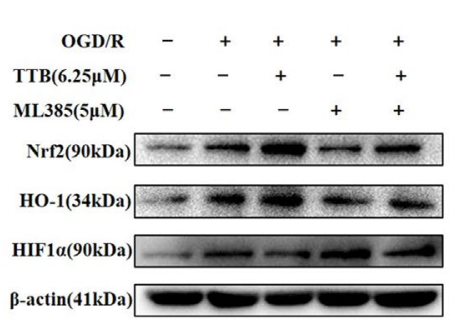

D
B

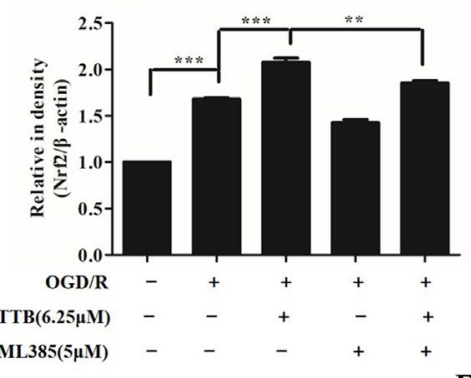

C

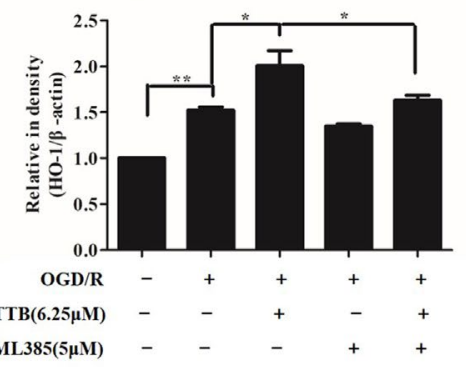

E

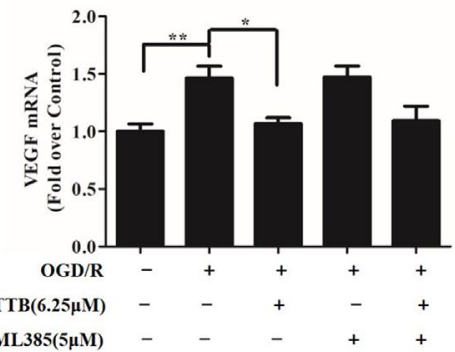

FIGURE 7 | Effects of ML385 (Nrf2-specific inhibitor) on Nrf2, HO-1, HIF-1 $\alpha$, and VEGF levels in OGD/R-treated astrocytes. Western blot and gPCR were carried out. Astrocytes were preincubated with ML385 at a concentration of $5 \mu \mathrm{M}$ for $12 \mathrm{~h}$ and then cells were treated with $6.25 \mu \mathrm{M} T \mathrm{~TB}$ for $6 \mathrm{~h}$ OGD and $24 \mathrm{~h}$ reoxygenation. (A-D) Expression of Nrf2, HO-1, and HIF-1 $\alpha$ was determined by Western blot, respectively. (E) Expression of VEGF in the mRNA level was determined by qPCR. ${ }^{*} p<0.05,{ }^{* *} p<0.01,{ }^{* \star *} p<0.001$. Data are mean \pm SE of three independent experiments. 
hydroxyl groups, which determines its significant antioxidant activity (Quiniou et al., 2008). Recent studies have identified that antioxidants can protect astrocytes from hypoxia/ischemia (H/I)-induced dysfunction (Bao et al., 2016). In the present study, we exposed primary cultures of astrocytes to OGD for $6 \mathrm{~h}$ and reoxygenation for $24 \mathrm{~h}$. Our results demonstrated for the first time that TTB at 6.25 and $25 \mu \mathrm{M}$ increased cell survival significantly in OGD/R-induced injury. TTB also decreased the $\mathrm{LDH}$ release to the culture medium.

$\mathrm{H} / \mathrm{I}$ exposure causes an oxidative stress and induces a significant damage in brain tissue, which can be described as an increase in the rate of ROS generation and imbalance of antioxidant defense system in the molecular level (Brekke et al., 2017; Parfenova et al., 2018). Excessive ROS initiates pro-inflammatory or growth stimulatory signals that are associated with cell death. Therefore, new pharmacological strategies aimed at the antioxidant system may potentially improve clinical management. The present study demonstrated that OGD/R exposure markedly increased ROS production in astrocytes compared to the control group and this increase was attenuated by TTB treatment. SODs, the antioxidant enzymes, are generally considered as $\mathrm{O}^{2-}$ scavengers against tissue and cellular damage caused by ROS (Fridovich, 1995; Li et al., 2008). In our study, OGD/R induced the decrease of SOD activity and TTB prevented the decrease of SOD activity in response to $\mathrm{OGD} / \mathrm{R}$. These data illustrated that TTB treatment had protective roles against $\mathrm{OGD} / \mathrm{R}$-induced oxidative stress.

$\mathrm{Nrf2}$ is a well-known key regulator of cellular resistance to oxidants and is activated through translocation from the cytoplasm to the nucleus, where it induces HO-1 gene expression as a target antioxidative gene (Kensler et al., 2007; Wu et al., 2015). HO-1, a rate-limiting enzyme in the transition of heme into biliverdin, also has a pivotal function in response to oxidative stress (Cao et al., 2017). When Nrf2 is up-regulated and translocated into the nucleus from the cytoplasm under stress conditions, the process is also essential for the activation of HO-1 expression (Kang et al., 2015). Growing evidence demonstrated that the $\mathrm{Nrf} 2 / \mathrm{HO}-1$ signaling pathway participated in the process of oxidative stress in several brain dysfunctional diseases (Meng et al., 2016; Bellaver et al., 2017; Zhao et al., 2018). Several antioxidant ingredients indicated that they protected cell damage by up-regulating Nrf2 and HO-1 expression in various diseases (Hsu et al., 2012; Yao et al., 2015; Jung et al., 2017). To explore whether TTB-induced cytoprotection was dependent on the presence of Nrf2 by inhibiting oxidative stress, the astrocytes were treated with OGD in different time courses of 6,12 , and $24 \mathrm{~h}$ followed by reoxygenation for $24 \mathrm{~h}$. Our results showed that the protein levels of Nrf2 were increased by OGD for $6 \mathrm{~h}$ and reoxygenation for $24 \mathrm{~h}$ but decreased by OGD for 12 or $24 \mathrm{~h}$ and reoxygenation for $24 \mathrm{~h}$ compared to the control group. Meanwhile, TTB up-regulated Nrf2 expression in total protein at all time points compared to the OGD/R group. The results demonstrated TTB potentially prolonged the activation state of the Nrf2 pathway and maintained the protection against oxidative stress in OGD/R-induced astrocytes. Furthermore, with OGD for 6 $\mathrm{h}$ and reoxygenation for $24 \mathrm{~h}$ treatment, TTB facilitated Nrf2 translocation to the nucleus and increased Nrf2 expression in the nucleus, suggesting that TTB promoted the activation of the $\mathrm{Nrf} 2 / \mathrm{HO}-1$ pathway in $\mathrm{H} / \mathrm{I}$ injury in the early phase.

HIF-1 $\alpha$ is an important transcription factor in a wide variety of responses to hypoxia (Chavez et al., 2000). Using the astrocyteneuron coculture model, the selective loss of HIF-1a function in neuron induced neuronal susceptibility to $\mathrm{H} / \mathrm{I}$ injury, whereas the loss of HIF-1 $\alpha$ function in astrocytes inhibits neuronal death by hypoxia (Vangeison et al., 2008). During hypoxia-induced CNS injury, HIF-1a expression targets multiple genes, including VEGF. The activation of VEGF expression under hypoxic conditions has been investigated in several studies. Notably, astrocytes secrete basal levels of VEGF under physiological conditions and the expression is further up-regulated by hypoxia. VEGF gene expression is transcriptionally regulated by HIF-1a (Marti et al., 2000; Schmid-Brunclik et al., 2008; Wiesner et al., 2013). Some previous studies identified that VEGF protects neurons from ischemic insults and promoted neurogenesis after cerebral ischemic injury (Ma et al., 2012; Liu et al., 2018). However, other studies reported that anti-VEGF treatment blocks vascular leakage in hypoxia (Nordal et al., 2004; Kaur et al., 2006). In the present study, it showed that OGD/R induced HIF-1 $\alpha$ and VEGF up-regulation. TTB inverted the effect of OGD/R on HIF-1a/VEGF expression in astrocytes. The results disclosed that the HIF-1 $\alpha$ /VEGF pathway might be involved in the astrocyte oxidative stress, providing new insights into TTB protection.

Nrf2 and HIF-1 $\alpha$ represent the oxygen and redox statedependent transcription factors. Their stabilization by redox status decides the cell fate, which means the existence of interplay between Nrf2 and the HIF-1a/VEGF signaling pathway under $\mathrm{H} / \mathrm{I}$ injury. One study demonstrated that hypoxia induced Nrf2 activation, resulting in the induction of Nrf2-dependent target thioredoxin-1 enhancement of HIF-1 $\alpha$ response in A549 cells (Malec et al., 2010). Li et al. indicated that Nrf2 knockdown inhibits venous hypertension-induced activation of the HIF-1a/ VEGF pathway (Li et al., 2016). In our study, TTB may act as an Nrf2 activator that up-regulated and maintained Nrf2 expression after OGD/R. To further explore whether the protection of TTB on OGD/R-induced injury in astrocytes was dependent on the activation of the Nrf2 pathway, ML385, a small-molecule Nrf2 inhibitor, was implemented to observe the protective mechanism of TTB. ML385 increases the ubiquitination and inhibits the proteasome degradation of Nrf2 binding to Keap1 and subsequently suppresses Nrf2 expression (Jung et al., 2018). Our results displayed that, with the combination of TTB and MLB385 treatment, MLB385 reversed the TTB-induced up-regulation of Nrf2 and HO-1 expression in OGD/R-induced astrocytes. These results indicated that the activation of the Nrf2/ HO-1 signaling pathway after TTB treatment was responsible for the protection of antioxidative stress. Furthermore, we found that, under OGD/R treatment in astrocytes, MLB385 induced the maintenance of the high level of HIF-1a expression. The combination of TTB and MLB385 decreased the HIF-1 $\alpha$ protein level compared to MLB385 alone. These results suggested that TTB inhibited OGD/R-induced astrocyte oxidative stress at least partially through the down-regulation of HIF-1 $\alpha$ and VEGF via the Nrf2/HO-1 signaling pathway. 
CNS diseases, such as trauma, $\mathrm{H} / \mathrm{I}$ injury, neuroinflammation, or neurodegeneration, cause astrocytes to become reactive. Reactive astrocytes were verified to control formation, maintenance, function, and the removal of neuronal synapses (Eroglu and Barres, 2010; Koizumi et al., 2018). Our study verified that $\mathrm{OGD} / \mathrm{R}$ induced astrocyte reactivation by up-regulating GFAP expression. Meanwhile, TTB inhibited GFAP expression, which revealed that TTB inhibited OGD/R-induced astrocyte reactivation. The mechanism might go through the alteration of factor secretion and gene expression. A previous study showed that proteins released by astrocytes selectively increased neuron axon length, branching, function, and synapse formation (Hughes et al., 2010). Other study demonstrated that astrocytes produced mRNAs that encoded synaptic adhesion proteins, which affected neuronal synapse formation (Cahoy et al., 2008). Astrocyte-neuron interaction might participate in neuronal plasticity. In our study, with neuron-astrocyte coculture, OGD/ R-induced astrocytes inhibited neurite growth in neurons compared to the control group. TTB-treated astrocytes reversed the inhibition of $\mathrm{OGD} / \mathrm{R}$ on neurite growth of neurons in the coculture system. It suggested that TTB regulated astrocyte function and subsequently promoted neuronal plasticity under $\mathrm{H} / \mathrm{I}$ injury. However, the deep mechanism of which factors were secreted by astrocytes and which genes were regulated remains unknown.

Taken together, TTB displays antioxidant activities in OGD/R-induced astrocytes. Our study provides evidence that TTB effectively suppresses excessive ROS production and increases SOD activity in terms of attenuation of HIF- $1 \alpha$ and VEGF expression by activating the Nrf2/HO-1 pathway, which depends on Nrf2 nuclear translocation and up-regulation of HO-1, to protect OGD/R-induced cell oxidative stress. Also, TTB administration in reactive astrocytes by OGD/R might contribute to reverse the inhibition of $\mathrm{OGD} / \mathrm{R}$ on neurite growth in neurons. These data suggest that TTB could be a novel

\section{REFERENCES}

Bao, G., Li, C., Qi, L., Wang, N., and He, B. (2016). Tetrandrine protects against oxygen-glucose-serum deprivation/reoxygenation-induced injury via PI3K/ AKT/NF-kappaB signaling pathway in rat spinal cord astrocytes. Biomed. Pharmacother. 84, 925-930. doi: 10.1016/j.biopha.2016.10.007

Bellaver, B., Souza, D. G., Souza, D. O., and Quincozes-Santos, A. (2017). Hippocampal astrocyte cultures from adult and aged rats reproduce changes in glial functionality observed in the aging brain. Mol. Neurobiol. 54, 2969-2985. doi: 10.1007/s12035-016-9880-8

Brekke, E., Berger, H. R., Wideroe, M., Sonnewald, U., and Morken, T. S. (2017). Glucose and intermediary metabolism and astrocyte-neuron interactions following neonatal hypoxia-ischemia in rat. Neurochem. Res. 42, 115-132. doi: 10.1007/s11064-016-2149-9

Cahoy, J. D., Emery, B., Kaushal, A., Foo, L. C., Zamanian, J. L., Christopherson, K. S., et al. (2008). A transcriptome database for astrocytes, neurons, and oligodendrocytes: a new resource for understanding brain development and function. J. Neurosci. 28, 264-278. doi: 10.1523/JNEUROSCI.4178-07.2008

Cao, S., Du, J., and Hei, Q. (2017). Lycium barbarum polysaccharide protects against neurotoxicity via the Nrf2-HO-1 pathway. Exp. Ther. Med. 14, 49194927. doi: 10.3892/etm.2017.5127

Cao, S., Chao, D., Zhou, H., Balboni, G., and Xia, Y. (2015). A novel mechanism for cytoprotection against hypoxic injury: delta-opioid receptor-mediated medication that imparts effective neuroprotection against NHIE to prevent cerebral oxidative stress-induced injury.

\section{DATA AVAILABILITY}

The datasets for this manuscript are not publicly available because all the data can be found in the manuscript. Requests to access the datasets should be directed to enjoyyz@163.com.

\section{ETHICS STATEMENT}

This study was carried out in accordance with the recommendations of the Medical College of Yangzhou University Guide for Care and Use of Laboratory Animals. The protocol was approved by the Committee of Care and Use of Laboratory of Medical College of Yangzhou University.

\section{AUTHOR CONTRIBUTIONS}

$\mathrm{XZ}$ and LL contributed to the design of the study. LL, ZZ, QY, and XZ performed the experiments. ZZ, LL, and XZ analyzed and interpreted the data. XZ, LL, and ZZ drafted and revised the manuscript. All the authors approved the final version of manuscript.

\section{FUNDING}

This study was supported by the National Natural Science Foundation of China (81701211 and 81703812), Traditional Chinese Medicine Bureau of Jiangsu Province Project (YB2015182), Jiangsu Pharmaceutical Association-Aosaikang Clinical Pharmacy Foundation (A201737), Qinglan Project of Yangzhou University (20180210), and "Summit of the Six Top Talents" Program of Jiangsu Province (WSN-277).

increase in Nrf2 translocation. Br. J. Pharmacol. 172, 1869-1881. doi: 10.1111/ bph.13031

Cengiz, P., Kintner, D. B., Chanana, V., Yuan, H., Akture, E., Kendigelen, P., et al. (2014). Sustained $\mathrm{Na}^{+} / \mathrm{H}^{+}$exchanger activation promotes gliotransmitter release from reactive hippocampal astrocytes following oxygen-glucose deprivation. PLoS One 9, e84294. doi: 10.1371/journal.pone.0084294

Chavez, J. C., Agani, F., Pichiule, P., and Lamanna, J. C. (2000). Expression of hypoxia-inducible factor-1alpha in the brain of rats during chronic hypoxia. J. Appl. Physiol. 89, 1937-1942. doi: 10.1152/jappl.2000.89.5.1937

Cui, X., Song, H., and Su, J. (2017). Curcumin attenuates hypoxic-ischemic brain injury in neonatal rats through induction of nuclear factor erythroid-2-related factor 2 and heme oxygenase-1. Exp. Ther. Med. 14, 1512-1518. doi: 10.3892/ etm. 2017.4683

Dilenge, M. E., Majnemer, A., and Shevell, M. I. (2001). Long-term developmental outcome of asphyxiated term neonates. J. Child Neurol. 16, 781-792. doi: 10.1177/08830738010160110201

Edwards, A. D., Brocklehurst, P., Gunn, A. J., Halliday, H., Juszczak, E., Levene, M., et al. (2010). Neurological outcomes at 18 months of age after moderate hypothermia for perinatal hypoxic ischaemic encephalopathy: synthesis and meta-analysis of trial data. BMJ 340, c363. doi: 10.1136/ bmj.c363

Eroglu, C., and Barres, B. A. (2010). Regulation of synaptic connectivity by glia. Nature 468, 223-231. doi: 10.1038/nature09612 
Fatemi, A., Wilson, M. A., and Johnston, M. V. (2009). Hypoxic-ischemic encephalopathy in the term infant. Clin. Perinatol. 36, 835-858. doi: 10.1016/j. clp.2009.07.011

Fridovich, I. (1995). Superoxide radical and superoxide dismutases. Annu. Rev. Biochem. 64, 97-112. doi: 10.1146/annurev.bi.64.070195.000525

Gao, Y., Fu, R., Wang, J., Yang, X., Wen, L., and Feng, J. (2018). Resveratrol mitigates the oxidative stress mediated by hypoxic-ischemic brain injury in neonatal rats via Nrf2/HO-1 pathway. Pharm. Biol. 56, 440-449. doi: 10.1080/13880209.2018.1502326

Guizzetti, M., Zhang, X., Goeke, C., and Gavin, D. P. (2014). Glia and neurodevelopment: focus on fetal alcohol spectrum disorders. Front. Pediatr. 2, 123. doi: 10.3389/fped.2014.00123

Hertz, L., Peng, L., and Lai, J. C. (1998). Functional studies in cultured astrocytes. Methods 16, 293-310. doi: 10.1006/meth.1998.0686

Hsu, Y. Y., Chen, C. S., Wu, S. N., Jong, Y. J., and Lo, Y. C. (2012). Berberine activates Nrf2 nuclear translocation and protects against oxidative damage via a phosphatidylinositol 3-kinase/Akt-dependent mechanism in NSC34 motor neuron-like cells. Eur. J. Pharm. Sci. 46, 415-425. doi: 10.1016/j.ejps.2012. 03.004

Hughes, E. G., Elmariah, S. B., and Balice-Gordon, R. J. (2010). Astrocyte secreted proteins selectively increase hippocampal GABAergic axon length, branching, and synaptogenesis. Mol. Cell. Neurosci. 43, 136-145. doi: 10.1016/j. mcn.2009.10.004

Jung, B. J., Yoo, H. S., Shin, S., Park, Y. J., and Jeon, S. M. (2018). Dysregulation of NRF2 in cancer: from molecular mechanisms to therapeutic opportunities. Biomol. Ther. (Seoul). 26, 57-68. doi: 10.4062/biomolther.2017.195

Jung, E., Kim, J. H., Kim, M. O., Lee, S. Y., and Lee, J. (2017). Melanocyte-protective effect of afzelin is mediated by the Nrf2-ARE signalling pathway via GSK-3beta inactivation. Exp. Dermatol. 26, 764-770. doi: 10.1111/exd.13277

Kang, J. S., Choi, I. W., Han, M. H., Kim, G. Y., Hong, S. H., Park, C., et al. (2015). The cytoprotective effects of 7,8-dihydroxyflavone against oxidative stress are mediated by the upregulation of Nrf2-dependent $\mathrm{HO}-1$ expression through the activation of the PI3K/Akt and ERK pathways in C2C12 myoblasts. Int. J. Mol. Med. 36, 501-510. doi: 10.3892/ijmm.2015.2256

Kaur, C., Sivakumar, V., Zhang, Y., and Ling, E. A. (2006). Hypoxia-induced astrocytic reaction and increased vascular permeability in the rat cerebellum. Glia 54, 826-839. doi: 10.1002/glia.20420

Kensler, T. W., Wakabayashi, N., and Biswal, S. (2007). Cell survival responses to environmental stresses via the Keap1-Nrf2-ARE pathway. Annu. Rev. Pharmacol. Toxicol. 47, 89-116. doi: 10.1146/annurev.pharmtox.46.120604.141046

Koizumi, S., Hirayama, Y., and Morizawa, Y. M. (2018). New roles of reactive astrocytes in the brain; an organizer of cerebral ischemia. Neurochem. Int. 119, 107-114. doi: 10.1016/j.neuint.2018.01.007

Kurinczuk, J. J., White-Koning, M., and Badawi, N. (2010). Epidemiology of neonatal encephalopathy and hypoxic-ischaemic encephalopathy. Early Hum. Dev. 86, 329-338. doi: 10.1016/j.earlhumdev.2010.05.010

Li, L., Pan, H., Wang, H., Li, X., Bu, X., Wang, Q., et al. (2016). Interplay between VEGF and Nrf2 regulates angiogenesis due to intracranial venous hypertension. Sci. Rep. 6, 37338. doi: 10.1038/srep37338

Li, Y., Bao, Y., Jiang, B., Wang, Z., Liu, Y., Zhang, C., et al. (2008). Catalpol protects primary cultured astrocytes from in vitro ischemia-induced damage. Int. Dev. Neurosci. 26, 309-317. doi: 10.1016/j.ijdevneu.2008.01.006

Liu, L., Li, J., Zeng, K. W., Jiang, Y., and Tu, P. F. (2016a). Five new biphenanthrenes from Cremastra appendiculata. Molecules 21, 1089-1098. doi: 10.3390/ molecules21081089

Liu, L., Yin, Q. M., Zhang, X. W., Wang, W., Dong, X. Y., Yan, X., et al. (2016b). Bioactivity-guided isolation of biphenanthrenes from Liparis nervosa. Fitoterapia 115, 15-18. doi: 10.1016/j.fitote.2016.09.006

Liu, M., Wu, Y., Liu, Y., Chen, Z., He, S., Zhang, H., et al. (2018). Basic fibroblast growth factor protects astrocytes against ischemia/reperfusion injury by upregulating the caveolin-1/VEGF signaling pathway. J. Mol. Neurosci. 64, 211-223. doi: 10.1007/s12031-017-1023-9

Ma, Y., Zechariah, A., Qu, Y., and Hermann, D. M. (2012). Effects of vascular endothelial growth factor in ischemic stroke. J. Neurosci. Res. 90, 1873-1882. doi: $10.1002 /$ jnr.23088

Malec, V., Gottschald, O. R., Li, S., Rose, F., Seeger, W., and Hanze, J. (2010). HIF1alpha signaling is augmented during intermittent hypoxia by induction of the
Nrf2 pathway in NOX1-expressing adenocarcinoma A549 cells. Free Radic. Biol. Med. 48, 1626-1635. doi: 10.1016/j.freeradbiomed.2010.03.008

Marti, H. J., Bernaudin, M., Bellail, A., Schoch, H., Euler, M., Petit, E., et al. (2000). Hypoxia-induced vascular endothelial growth factor expression precedes neovascularization after cerebral ischemia. Am. J. Pathol. 156, 965-976. doi: 10.1016/S0002-9440(10)64964-4

Meng, X. E., Zhang, Y., Li, N., Fan, D. F., Yang, C., Li, H., et al. (2016). Effects of hyperbaric oxygen on the Nrf2 signaling pathway in secondary injury following traumatic brain injury. Genet. Mol. Res. 15, gmr6933. doi: 10.4238/ gmr.15016933

Mulkey, S. B., Fontenot, E. E., Imamura, M., and Yap, V. L. (2011). Therapeutic hypothermia in a neonate with perinatal asphyxia and transposition of the great arteries. Ther. Hypothermia Temp. Manag. 1, 205-208. doi: 10.1089/ther.2011.0016

Nelson, K. B. (2007). Perinatal ischemic stroke. Stroke 38, 742-745. doi: 10.1161/01. STR.0000247921.97794.5e

Nordal, R. A., Nagy, A., Pintilie, M., and Wong, C. S. (2004). Hypoxia and hypoxiainducible factor- 1 target genes in central nervous system radiation injury: a role for vascular endothelial growth factor. Clin. Cancer Res. 10, 3342-3353. doi: 10.1158/1078-0432.CCR-03-0426

Parfenova, H., Pourcyrous, M., Fedinec, A. L., Liu, J., Basuroy, S., and Leffler, C. W. (2018). Astrocyte-produced carbon monoxide and the carbon monoxide donor CORM-A1 protect against cerebrovascular dysfunction caused by prolonged neonatal asphyxia. Am. J. Physiol. Heart Circ. Physiol. 315, H978-H988. doi: 10.1152/ajpheart.00140.2018

Quiniou, C., Kooli, E., Joyal, J. S., Sapieha, P., Sennlaub, F., Lahaie, I., et al. (2008). Interleukin-1 and ischemic brain injury in the newborn: development of a small molecule inhibitor of IL-1 receptor. Semin. Perinatol. 32, 325-333. doi: 10.1053/j.semperi.2008.07.001

Sandberg, M., Patil, J., D’Angelo, B., Weber, S. G., and Mallard, C. (2014). NRF2regulation in brain health and disease: implication of cerebral inflammation. Neuropharmacology 79, 298-306. doi: 10.1016/j.neuropharm.2013.11.004

Schmid-Brunclik, N., Burgi-Taboada, C., Antoniou, X., Gassmann, M., and Ogunshola, O. O. (2008). Astrocyte responses to injury: VEGF simultaneously modulates cell death and proliferation. Am. J. Physiol. Regul. Integr. Comp. Physiol. 295, R864-873. doi: 10.1152/ajpregu.00536.2007

Shu, L., Wang, C., Wang, J., Zhang, Y., Zhang, X., Yang, Y., et al. (2016). The neuroprotection of hypoxic preconditioning on rat brain against traumatic brain injury by up-regulated transcription factor Nrf2 and HO-1 expression. Neurosci. Lett. 611, 74-80. doi: 10.1016/j.neulet.2015.11.012

Tasca, C. I., Dal-Cim, T., and Cimarosti, H. (2015). In vitro oxygen-glucose deprivation to study ischemic cell death. Methods Mol. Biol. 1254, 197-210. doi: 10.1007/978-1-4939-2152-2_15

Ullian, E. M., Sapperstein, S. K., Christopherson, K. S., and Barres, B. A. (2001). Control of synapse number by glia. Science 291, 657-661. doi: 10.1126/science. 291.5504.657

Vangeison, G., Carr, D., Federoff, H. J., and Rempe, D. A. (2008). The good, the bad, and the cell type-specific roles of hypoxia inducible factor-lalpha in neurons and astrocytes. J. Neurosci. 28, 1988-1993. doi: 10.1523/JNEUROSCI. 5323-07.2008

Wiesner, D., Merdian, I., Lewerenz, J., Ludolph, A. C., Dupuis, L., and Witting, A. (2013). Fumaric acid esters stimulate astrocytic VEGF expression through HIF-1alpha and Nrf2. PLoS One 8, e76670. doi: 10.1371/journal.pone.0076670

Wu, L., Li, H. H., Wu, Q., Miao, S., Liu, Z. J., Wu, P., et al. (2015). Lipoxin $\mathrm{A}_{4}$ activates Nrf2 pathway and ameliorates cell damage in cultured cortical astrocytes exposed to oxygen-glucose deprivation/reperfusion insults. J. Mol. Neurosci. 56, 848-857. doi: 10.1007/s12031-015-0525-6

Xue, Z., Li, S., Wang, S., Wang, Y., Yang, Y., Shi, J., et al. (2006). Mono-, bi-, and triphenanthrenes from the tubers of Cremastra appendiculata. J. Nat. Prod. 69, 907-913. doi: 10.1021/np060087n

Yao, J., Zhang, B., Ge, C., Peng, S., and Fang, J. (2015). Xanthohumol, a polyphenol chalcone present in hops, activating Nrf2 enzymes to confer protection against oxidative damage in PC12 cells. J. Agric. Food Chem. 63, 1521-1531. doi: $10.1021 /$ jf505075n

Zalewska, T., Jaworska, J., and Ziemka-Nalecz, M. (2015). Current and experimental pharmacological approaches in neonatal hypoxic-ischemic encephalopathy. Curr. Pharm. Des. 21, 1433-1439. doi: 10.2174/1381612820 999141029162457 
Zhang, X., Bhattacharyya, S., Kusumo, H., Goodlett, C. R., Tobacman, J. K., and Guizzetti, M. (2014). Arylsulfatase B modulates neurite outgrowth via astrocyte chondroitin-4-sulfate: dysregulation by ethanol. Glia 62 , 259-271. doi: $10.1002 /$ glia.22604

Zhao, Y. J., Nai, Y., Ma, Q. S., Song, D. J., Ma, Y. B., Zhang, L. H., et al. (2018). Dl-3-n-butylphthalide protects the blood brain barrier of cerebral infarction by activating the Nrf2/HO-1 signaling pathway in mice. Eur. Rev. Med. Pharmacol. Sci. 22, 2109-2118. doi: 10.26355/eurrev20180414744

Zorec, R., Parpura, V., and Verkhratsky, A. (2018). Astroglial vesicular network: evolutionary trends, physiology and pathophysiology. Acta Physiol. (Oxf.) 222, e12915. doi: 10.1111/apha.12915
Conflict of Interest Statement: The authors declare that the research was conducted in the absence of any commercial or financial relationships that could be construed as a potential conflict of interest.

Copyright (c) $2019 \mathrm{Liu}$, Zhao, Yin and Zhang. This is an open-access article distributed under the terms of the Creative Commons Attribution License (CC $B Y)$. The use, distribution or reproduction in other forums is permitted, provided the original author(s) and the copyright owner(s) are credited and that the original publication in this journal is cited, in accordance with accepted academic practice. No use, distribution or reproduction is permitted which does not comply with these terms. 